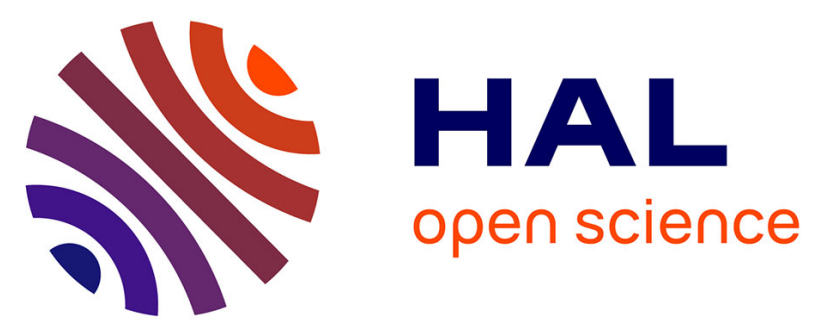

\title{
A Closed-Form Solution to the Power Minimization Problem over Two Orthogonal Frequency Bands under QoS and Cognitive Radio Interference Constraints
} Raouia Masmoudi, Elena Veronica Belmega, Inbar Fijalkow, Noura Sellami

\section{- To cite this version:}

Raouia Masmoudi, Elena Veronica Belmega, Inbar Fijalkow, Noura Sellami. A Closed-Form Solution to the Power Minimization Problem over Two Orthogonal Frequency Bands under QoS and Cognitive Radio Interference Constraints. IEEE DySPAN 2012, Oct 2012, Bellevue, United States. pp.11. hal-00736086

\author{
HAL Id: hal-00736086 \\ https://hal.science/hal-00736086
}

Submitted on 27 Sep 2012

HAL is a multi-disciplinary open access archive for the deposit and dissemination of scientific research documents, whether they are published or not. The documents may come from teaching and research institutions in France or abroad, or from public or private research centers.
L'archive ouverte pluridisciplinaire HAL, est destinée au dépôt et à la diffusion de documents scientifiques de niveau recherche, publiés ou non, émanant des établissements d'enseignement et de recherche français ou étrangers, des laboratoires publics ou privés. 


\title{
A Closed-Form Solution to the Power Minimization Problem over Two Orthogonal Frequency Bands under QoS and Cognitive Radio Interference Constraints
}

\author{
Raouia Masmoudi ${ }^{\ddagger}$, E. Veronica Belmega ${ }^{\dagger}$, Inbar Fijalkow ${ }^{\dagger}$, Noura Sellami ${ }^{\ddagger}$ \\ $\dagger$ ETIS/ ENSEA, Université Cergy-Pontoise, CNRS \\ 6 Avenue du Ponceau \\ F-95000 Cergy-Pontoise, France \\ $\ddagger$ LETI, ENIS and University of Sfax \\ 3038, Sfax, Tunisia \\ Email:\{raouia.masmoudi, belmega, inbar.fijalkow\}@ensea.fr, sellami_noura@yahoo.fr
}

\begin{abstract}
This paper considers a Cognitive Radio (CR) channel composed of a secondary user (SU) and a primary user (PU). An analysis of the power minimization over several orthogonal frequency bands at the SU level under the following constraints is provided: a minimum Quality of Service (QoS) constraint, maximum peak and average interference to the PU constraints. The general solution, when it exists, is a water-filling type of solution which can be computed via iterative algorithms. It turns out that, in the case of two orthogonal bands a closed-form analytical solution can be found and a complete analysis of the feasibility of these opposing constraints is presented in details. Several numerical results that sustain and give inside into the analysis are also discussed.
\end{abstract}

Index Terms-Cognitive Radio channels, power-efficient spectrum allocation problems, green communications

\section{INTRODUCTION}

The concept of Cognitive Radio [1] has recently emerged as a promising paradigm for a more efficient use of the available spectrum by allowing the coexistence of licensed (primary) and unlicensed (secondary) users in the same bandwidth. Another major concern that is rapidly gaining momentum is the energy consumption for green communications. Recently, there has been an important switch of focus from a data rate maximization vision to an application-based utility optimization one, that takes into account the cost of power consumption to achieve the target data rates [2], [3].

In this paper, we consider a home-automation scenario where several technologies (i.e., WiFi, PLC, Femto, etc.) are able to operate simultaneously and where different appliances may have different hierarchical priorities. The EconHome-box is meant to allocate frequency bands and powers in a green telecommunications sprint. Notice that the analysis we provide is not restricted to this scenario alone and can be applied to any CR system with several orthogonal sub-channels available

This work was sponsored in part by the FUI SYSTEM@TIC Project EconHome. for communication: OFDM systems, interoperability of several coexisting transmission systems.

To model this home-automation scenario we consider a CR channel composed of a primary user (PU) and a secondary user (SU) that is opportunistically accessing the channel and interfering with the PU. The question under investigation is to know how the SU will optimally allocate the available power over the available bands to minimize its total power consumption under several constraints: target QoS constraint, peak and average maximal interference levels that the SU can create to the PU.

The problem of resource allocation has mainly been studied from a data rate maximization point of view. For exemple, in [4], [5], [6] the authors considered the maximization of the Shannon achievable rate over several sub-channels, subject to transmit powers and mask constraints on each sub-channel. In [4], a centralized approach based on convex optimization (see e.g., [7], [8]) was proposed to compute, under some technical conditions, the achievable rate region of the system.

To the best of the authors' knowledge, the closest works to ours are [9] and [10]. In [10], the authors consider the distributed minimization of transmit powers subject to rate target constraints for the multi-user parallel interference channels. The difference to our work consists in the absence of PU and thus, of the interference constraints that limit the SU transmissions'. In [9], the authors study the multiusers multiple-input multiple-output (MIMO) Cognitive Radio channels. In order to design cognitive MIMO SU transceivers, the authors of [9] model the distributed maximization of the Shannon achievable rates under interference constraints imposed by the presence of the PU. In order to approach this complex problem, the authors study several particular cases by systematically eliminating one of the interference constraints and solving a simpler problem. In this paper, we consider a simpler channel model (the single-SU case and the multiple 
orthogonal sub-channels). However, the novelty and interest of our work consists in the fact that we study the dual problem of [9] in its generality with respect to the feasibility constraints.

Our contributions can be summarized as follows: i) in the general case of arbitrary number of orthogonal bands, we give the analytical water-filling solution to the power minimization problem under minimum rate and maximum peak and average interference constraints created to the PU, when it exists; ii) in the case of two orthogonal frequency bands, we give an analytical closed-form solution and a complete study of the feasibility of all the constraints is provided; iii) numerical simulations that sustain the analysis are provided for different channel parameters.

The remainder of this paper is organized as follows. In section II, we describe the system model. We provide the water-filling type of solution of the general optimization problem section III. A closed-form solution in the case of two orthogonal bands is given in section IV. In section V, we present some interesting simulation results. We conclude this paper in sectionVI.

\section{System ModeL}

The CR channel under study system is depicted in Fig.1. The primary/secondary user consists of a Primary/Secondary Transmitter (PT/ST) and a Primary/Secondary Receiver (PR/ST) respectively. Each transmitter or receiver is equipped with only one antenna. The transmission is done over $N \geq 2$ orthogonal frequency bands. The transmit power of ST in the frequency band $k \in\{1, \ldots N\}$ is denoted by $p_{k}$. We denote the overall power allocation profile by $p=\left(p_{1}, p_{2}, \ldots, p_{N}\right)$, $p \in \mathbb{R}_{+}^{N}$.

The received signal at $\mathrm{SR}$ in band $k$ can be written as:

$$
y_{k}=\sqrt{p_{k}} h_{k} s_{k}+i_{k, P U}+n_{k},
$$

where $h_{k}$ represents the channel gain, $s_{k}$ the transmit signal of the ST is given by unit-power variable over each orthogonal frequency band. The instantaneous power gain of the STSR direct link, the interfering ST-PR link is denoted by are denoted by $\left|h_{k}\right|^{2}, g_{k}$ respectively. All the channels are assumed to be stationary, ergodic and independent from the noises. The noise $n_{k} \backsim \mathcal{N}\left(0, \sigma_{k}^{2}\right)$ is a zero-mean circularly symmetric complex Gaussian noise vector and the interfering signal from the PU $i_{k, P U} \backsim \mathcal{N}\left(0, \sigma_{k, P U}^{2}\right)$.

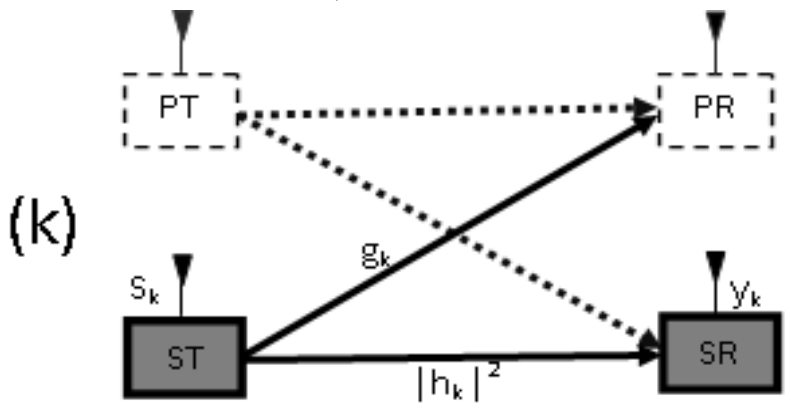

Fig. 1. Channel model for the communication in band $k$.
In this scenario, the Shannon achievable rate of the SU is given by

$$
R(\underline{p})=\sum_{k=1}^{N} \log _{2}\left(1+c_{k} p_{k}\right),
$$

where $c_{k}$ is related to the interfering ST-PR link by

$$
c_{k}=\frac{\left|h_{k}\right|^{2}}{\sigma_{k}^{2}+\sigma_{k, P U}^{2}}
$$

where $\sigma_{k, P U}^{2}$ is the interfering variance from the PU and $\sigma_{k}^{2}$ is the variance of the thermal noise.

\section{GENERAL OPTIMIZATION PROBLEM}

We consider the constrained power minimization problem at the SU level. Three constraints are considered in our optimization problem: a) a QoS constraint (4); b) peak (6) and average (5) interference constraints to protect the PU and limit the interference caused by the SU.

1- Target rate constraint:

$$
R(\underline{p})=\sum_{k=1}^{N} \log _{2}\left(1+c_{k} p_{k}\right) \geq R_{\text {min }}
$$

which is imposed to achieve a minimum $\mathrm{QoS}$ of the SU transmission.

2- Average interference power shaping constraint:

$$
\sum_{k=1}^{N} g_{k} p_{k} \leq \bar{P},
$$

where $\bar{P}$ is the maximum average interference level that can be received at PR. This constraint is an imposed interference limitation at the PR.

3- Peak interference power shaping constraints:

$$
0 \leq g_{k} p_{k} \leq P_{k}^{p e a k}, \quad \forall k=1, \ldots N,
$$

where $P_{k}^{\text {peak }}$ is the maximum peak interference level that can be received at PR in a given band.

The optimization problem under study can be written as:

$$
\begin{cases}\min _{\left\{p_{k}\right\}_{k=1}^{N}} \sum_{k=1}^{N} p_{k} \\ \text { s.t. } & R(\underline{p})=\sum_{k=1}^{N} \log _{2}\left(1+c_{k} p_{k}\right) \geq R_{\min } \\ & \sum_{k=1}^{N} g_{k} p_{k} \leq \bar{P} \\ & 0 \leq g_{k} p_{k} \leq P_{k}^{\text {peak }} \quad \forall k \in\{1, . . N\}\end{cases}
$$

Notice that without loss of generality, we can consider only the case where $R_{\text {min }}>0$. In the case where $R_{\text {min }}=0$ the solution is trivial and the transmission powers are equal to zero, $p_{k}^{*}=0 \forall k$, i.e.,no transmission is performed at the $\mathrm{SU}$. 
In the case of applying a flat power allocation policy (i.e., $\forall k \in\{1, . . N\} p_{k}=p$ ), all the constraints become more strict and the feasible set is reduced. The constraints in this case will be given by $p \leq \min \left\{\frac{P_{k}^{\text {peak }}}{g_{k}}, \frac{\bar{P}}{\sum_{k} g_{k}}\right\}$ and $\sum_{k} \log \left(1+c_{k} p\right) \geq R_{\min }$. Therefore, the benefit of applying our optimal allocation policy comparing to this flat power allocation policy, is to have much more flexibility in the feasibility of our constraints and a significant gain in terms of power consumption.

Theorem 1: If the feasible set of the optimization problem (7) is not void, the optimal power allocation policy is given by the following water-filling type solution ${ }^{1}$ :

$$
p_{n}^{*}=\left[\xi_{n}-\frac{1}{c_{n}}\right]_{0}^{\frac{P_{n}^{\text {peak }}}{g_{n}}}, \forall n \in\{1, \ldots N\}
$$

where $\xi_{n}=\frac{\lambda}{\ln (2)\left(1+\beta g_{n}\right)}, \lambda$ and $\beta$ verify the following conditions:

$$
\left\{\lambda>0 \text { and } \sum_{k=1}^{N} \log _{2}\left(1+c_{k} p_{k}^{*}\right)=R_{\text {min }}\right\}
$$

and

$$
\left\{\beta>0 \text { and } \sum_{k=1}^{N} g_{k} p_{k}^{*}=\bar{P}\right\} \text { or }\left\{\beta=0 \text { and } \sum_{k=1}^{N} g_{k} p_{k}^{*}<\bar{P}\right\} \text {. }
$$

If the feasible set is void then the problem (7) has no solution.

Proof: We denote the feasible set by $\mathcal{S}_{N}$ such that:

$$
\begin{array}{r}
\mathcal{S}_{N}=\left\{\underline{p} \in \mathbb{R}_{+}^{N} \mid 0 \leq g_{k} p_{k} \leq P_{k}^{\text {peak }},\right. \\
\left.\sum_{k=1}^{N} \log _{2}\left(1+c_{k} p_{k}\right) \geq R_{\text {min }}, \sum_{k=1}^{N} g_{k} p_{k} \leq \bar{P}\right\} .
\end{array}
$$

Two different scenarios are possible:

- $\mathcal{S}_{N}=\varnothing$ : In this case, there is no feasible power allocation policy, i.e., no vector $\underline{p} \in \mathbb{R}_{+}^{N}$ that satisfies simultaneously all the constraints in (9).

- $\mathcal{S}_{N} \neq \varnothing$ : Given the concavity of $\log ($.$) function, the$ linearity of powers constraints and the convexity of the

${ }^{1}$ We denote by $[x]_{a}^{b}=\min \{\max \{x, a\}, b\}$ or equivalently

$$
[x]_{a}^{b}=\left\{\begin{array}{lll}
x, & \text { if } & a \leq x \leq b \\
a, & \text { if } & x<a \\
b, & \text { if } & x>b
\end{array}\right.
$$

objective function $\sum_{k=1}^{N} p_{k}$, it is easy to prove that $\mathcal{S}_{N}$ is convex [8], i.e., $\forall \widehat{p}_{k}, \tilde{p}_{k} \in \mathcal{S}_{N}, \quad \forall \theta \in[0,1]$ we have

$$
\theta \widehat{p}_{k}+(1-\theta) \tilde{p}_{k} \in \mathcal{S}_{N} .
$$

Thus, the optimization problem in (7) is a convex problem which has at least an optimal solution. Therefore, we can apply the Lagrangian method to solve (7).

The Lagrangian is given by:

$$
\begin{aligned}
& \mathcal{L}(\underline{p}, \lambda, \beta, \underline{\alpha}, \underline{\mu})=\sum_{k=1}^{N} p_{k}-\lambda\left(\sum_{k=1}^{N} \log _{2}\left(1+c_{k} p_{k}\right)\right. \\
& \left.-R_{\text {min }}\right)+\beta\left(\sum_{k=1}^{N} g_{k} p_{k}-\bar{P}\right)-\sum_{k=1}^{N} \alpha_{k} g_{k} p_{k} \\
& +\sum_{k=1}^{N} \mu_{k}\left(g_{k} p_{k}-P_{k}^{\text {peak }}\right),
\end{aligned}
$$

where $\lambda, \quad \beta, \quad \underline{\alpha}=\left(\alpha_{1}, \alpha_{2}, \ldots, \alpha_{N}\right)$ and $\underline{\mu}=$ $\left(\mu_{1}, \mu_{2}, \ldots, \mu_{N}\right)$ are the non-negative Lagrangian multipliers of the corresponding constraints (4)(5)(6), respectively. We denote $p^{*}$ an optimal solution.

The Karush-Kuhn-Tucker (KKT) optimality conditions are:

i) Compute the solution that gives null Lagrangian derivative: $\left.\frac{\partial \mathcal{L}}{\partial p_{n}}\right|_{p_{n}=p_{n}^{*}}=0$

ii) Non-negative Lagrangian multiplier of the rate constraint $\lambda>0$ and $\sum_{k=1}^{N} \log _{2}\left(1+c_{k} p_{k}^{*}\right)=R_{\text {min }}$
or $\lambda=0$ and $\sum_{k=1}^{N} \log _{2}\left(1+c_{k} p_{k}^{*}\right)>R_{\text {min }}$

The case of null Lagrangian multiplier of the rate constraint cannot occur, because if $\lambda=0$ then the Lagrangian in (11) becomes:

$$
\begin{gathered}
\mathcal{L}(\underline{p}, \beta, \underline{\alpha}, \underline{\mu})=\sum_{k=1}^{N} p_{k}+\beta\left(\sum_{k=1}^{N} g_{k} p_{k}-\bar{P}\right) \\
-\sum_{k=1}^{N} \alpha_{k} g_{k} p_{k}+\sum_{k=1}^{N} \mu_{k}\left(g_{k} p_{k}-P_{k}^{\text {peak }}\right),
\end{gathered}
$$

and the optimization problem in (7) is given by:

$$
\begin{cases}\min _{\left\{p_{k}\right\}_{k=1}^{N}} & \sum_{k=1}^{N} p_{k} \\ \text { s.t. } & \sum_{k=1}^{N} g_{k} p_{k} \leq \bar{P} \\ & 0 \leq g_{k} p_{k} \leq P_{k}^{\text {peak }} \quad \forall k \in\{1, . . N\}\end{cases}
$$

In this power optimization problem the optimal power solution $\underline{p}^{*}$ is null. Then the SU optimal rate is null also (i.e., $\sum_{k=1}^{N} \log _{2}\left(1+c_{k} p_{k}^{*}\right)=0$ ), this is 
contradictory to the QoS constraint which is strictly positive $\left(R_{\min }>0\right)$. Thus, the Lagrangian multiplier of the rate constraint is strictly positive and given by: $\lambda>0$ and $\sum_{k=1}^{N} \log _{2}\left(1+c_{k} p_{k}^{*}\right)=R_{\text {min }}$

iii) Non-negative Lagrangian multiplier of the average interference power constraint $\beta>0$ and $\sum_{k=1}^{N} g_{k} p_{k}^{*}=$ $\bar{P}$ or $\beta=0$ and $\sum_{k=1}^{N} g_{k} p_{k}^{*}<\bar{P} ;$

iv) Non-negative Lagrangian multiplier of the peak powers constraints for each band:

$$
\begin{gathered}
\alpha_{n}>0 \text { and } g_{n} p_{n}^{*}=0 \text { or } \alpha_{n}=0 \text { and } g_{n} p_{n}^{*}>0 \\
\mu_{n}>0 \text { and } p_{n}^{*}=\frac{P_{n}^{\text {peak }}}{g_{n}} \text { or } \mu_{n}=0 \text { and } p_{n}^{*}<\frac{P_{n}^{\text {peak }}}{g_{n}} .
\end{gathered}
$$

As we said before, in order to compute the optimal solution of our problem we derive the Lagrangian in (11),

$$
\begin{aligned}
& \left.\frac{\partial \mathcal{L}}{\partial p_{n}}\right|_{p_{n}=p_{n}^{*}}=0, \\
\Leftrightarrow & 1-\frac{\lambda c_{n}}{\ln (2)\left(1+c_{n} p_{n}^{*}\right)}+\left(\beta-\alpha_{n}+\mu_{n}\right) g_{n}=0, \\
\Leftrightarrow & \frac{\lambda c_{n}}{\ln (2)\left(1+c_{n} p_{n}^{*}\right)}=1+\left(\beta-\alpha_{n}+\mu_{n}\right) g_{n}, \\
\Leftrightarrow & \left(1+c_{n} p_{n}^{*}\right)=\frac{\lambda c_{n}}{\ln (2)\left(1+\left(\beta-\alpha_{n}+\mu_{n}\right) g_{n}\right)}, \\
\Leftrightarrow & p_{n}^{*}=\frac{\lambda}{\ln (2)\left(1+\left(\beta-\alpha_{n}+\mu_{n}\right) g_{n}\right)}-\frac{1}{c_{n}} .
\end{aligned}
$$

Considering the other KKT conditions, the solution in function of the optimal $\left(\alpha_{n}, \mu_{n}\right)$ is as follows:

$\star \alpha_{n}=0$ and $\mu_{n}=0$ (the optimal power solution is strictly ${ }^{2}$ between 0 and $P_{n}^{\text {peak }} / g_{n}$ )

$$
p_{n}^{*}=\left(\frac{\lambda}{\ln (2)\left(1+\beta g_{n}\right)}-\frac{1}{c_{n}}\right)_{0}^{\frac{P_{n}^{\text {peak }}}{g_{n}}},
$$

$\star \alpha_{n}=0$ and $\mu_{n}>0$,

$$
p_{n}^{*}=\frac{P_{n}^{\text {peak }}}{g_{n}},
$$

${ }^{2}$ The notation $(x)_{a}^{b}$ is defined by:

$$
(x)_{a}^{b}=\left\{\begin{array}{lc}
x, & \text { if } a<x<b \\
0, & \text { otherwise. }
\end{array}\right.
$$

$$
\begin{aligned}
& \star \alpha_{n}>0 \text { and } \mu_{n}=0, \\
& \qquad p_{n}^{*}=0 .
\end{aligned}
$$

Finally, the equations (12), (13) and (14) can be re-written in a compact form as follows:

$$
p_{n}^{*}=\left[\frac{\lambda}{\ln (2)\left(1+\beta g_{n}\right)}-\frac{1}{c_{n}}\right]_{0}^{\frac{P_{n}^{\text {peak }}}{g_{n}}},
$$

where $\lambda$ and $\beta$ verify the following conditions:

$$
\begin{gathered}
\left\{\lambda>0 \text { and } \sum_{k=1}^{N} \log _{2}\left(1+c_{k} p_{k}^{*}\right)=R_{\text {min }}\right\} \\
\left\{\beta>0 \text { and } \sum_{k=1}^{N} g_{k} p_{k}^{*}=\bar{P}\right\} \\
\text { or }\left\{\beta=0 \text { and } \sum_{k=1}^{N} g_{k} p_{k}^{*}<\bar{P}\right\} .
\end{gathered}
$$

Thus, by making a change of variable $\xi_{n}=\frac{\lambda}{\ln (2)\left(1+\beta g_{n}\right)}$, we obtain the analytical solution of the problem (7), which is expressed by the well known water-filling:

$$
p_{n}^{*}=\left[\xi_{n}-\frac{1}{c_{n}}\right]_{0}^{\frac{P_{n}^{\text {peak }}}{g_{n}}} .
$$

When the feasible set is void, one or more constraints need to be relaxed if the system owner wishes to allow a non-trivial secondary transmission.

\section{THE CASE OF TWO ORTHOGONAL FREQUENCY BANDS}

In this section, we focus on the specific case where only two orthogonal frequency bands are available, i.e., $N=2$. This is motivated by the fact that an analytical study of the feasible set in the general scenario (9) is very difficult. As we will show, this difficulty is overcome in this case. We provide a complete and closed-form analysis of the feasibility of the opposing constraints: the QoS constraint on one hand, and the power constraints on the other. Moreover, this two bands case models the systems where two different technologies can coexist, i.e., (WiFi+PLC), or (WiFi+Femto), which is pertinent in current home-automation systems. Because these technologies are inherently different or heterogeneous, it is realistic to assume the orthogonality property of the sub-channels.

In what follows, we focus on the simpler case of $N=2$ for the following reasons: a) it can be realistic in several scenarios among which we have mentioned here above two of them; b) it can be solved in an analytical closed-form way; c) it gives insight and intuition on what happens in a general system with $N>2$ sub-channels which can be solved only numerically. 
In this case, the optimization problem in (7) can be reformulated as:

$$
\begin{gathered}
\min \left\{p_{1}+p_{2}\right\} \\
\text { s.t } \underline{p} \in \mathcal{S}_{2},
\end{gathered}
$$

where, the feasible set $\mathcal{S}_{2}$ is given by

$$
\begin{aligned}
& \mathcal{S}_{2}=\left\{\underline{p} \in \mathbb{R}_{+}^{2} \mid 0 \leq g_{1} p_{1} \leq P_{1}^{\text {peak }}, 0 \leq g_{2} p_{2} \leq P_{2}^{\text {peak }},\right. \\
& \left.\sum_{k=1}^{2} \log _{2}\left(1+c_{k} p_{k}\right) \geq R_{\text {min }}, \sum_{k=1}^{2} g_{k} p_{k} \leq \bar{P}\right\} .
\end{aligned}
$$

Theorem 2: In the case of two orthogonal frequency bands, when a solution to (17) exists, it is unique and its closed-form solution is given here under.

If $\bar{P}<-\frac{g_{1}}{c_{1}}-\frac{g_{2}}{c_{2}}+2 \sqrt{\left(\frac{g_{1}}{c_{1}} \frac{g_{2}}{c_{2}}\right) \exp \left(R_{\text {min }}\right)}$ or otherwise if $\max \left\{\widehat{p_{1}}, \widetilde{p_{1}}\right\}<0$ then the constraints (4) and (5) can not be simultaneously satisfied. Also if at least one of the following conditions is met: $\frac{P_{1}^{\text {peak }}}{g_{1}}<\widehat{p_{1}}$ or $\frac{P_{2}^{\text {peak }}}{g_{1}}<\min \left\{\widehat{p_{2}}, \tilde{p_{2}}\right\}$ or $\left\{f_{1}\left(\frac{P_{1}^{\text {peak }}}{g_{1}}\right)>P_{2}^{\text {peak }}\right.$ and $\left.f_{1}^{-1}\left(\frac{P_{2}^{\text {peak }}}{g_{2}}\right)>\frac{P_{1}^{\text {peak }}}{g_{1}}\right\}$ then constraint (6) can not be satisfied. In all the aforementioned cases, a feasible solution does not exist. In all other possible scenarios an optimal solution exists and is given by

$$
\left\{\begin{array}{l}
p_{1}^{*}=\left[\frac{-1}{c_{1}}+\sqrt{\left.\frac{\exp \left(R_{\text {min }}\right)}{c_{1} c_{2}}\right]_{x}^{y},}\right. \\
p_{2}^{*}=\left[\frac{-1}{c_{2}}+\sqrt{\frac{\exp \left(R_{\text {min }}\right)}{c_{1} c_{2}}}\right]_{w}^{z},
\end{array}\right.
$$

where $x, y, w, z$ are given by

$$
\left\{\begin{array}{l}
x=\max \left\{\max \left\{\widehat{p_{1}}, 0\right\}, f_{1}^{-1}\left(\frac{P_{2}^{\text {peak }}}{g_{2}}\right)\right\}, \\
y=\min \left\{\min \left\{\widetilde{p_{1}}, f_{1}^{-1}(0)\right\}, \frac{P_{1}^{\text {peak }}}{g_{1}}\right\}, \\
w=\max \left\{\max \left\{\widetilde{p_{2}}, 0\right\}, f_{1}\left(\frac{P_{1}^{\text {peak }}}{g_{1}}\right)\right\}, \\
z=\min \left\{\min \left\{\widehat{p_{2}}, f_{1}(0)\right\}, \frac{P_{2}^{\text {peak }}}{g_{2}}\right\} .
\end{array}\right.
$$

The parameters $\widehat{p}, \tilde{p}$ are the intersection points of the functions $f_{1}($.$) and \bar{f}_{2}($.$) defined as: f_{1}: \mathbb{R} \rightarrow \mathbb{R}$ (derived from the rate constraint(4)) such that:

$$
f_{1}\left(p_{1}\right)=\frac{1}{c_{2}}\left(\frac{\exp \left(R_{\min }\right)}{1+c_{1} p_{1}}-1\right)
$$

and $f_{2}: \mathbb{R} \rightarrow \mathbb{R}$ (derived from the average interference power constraint(5)) such that:

$$
f_{2}\left(p_{1}\right)=\frac{\bar{P}-p_{1} g_{1}}{g_{2}} .
$$

Proof:

In order to solve the optimization problem (17), we rewrite the feasible set using the two functions defined in (19) and (20) in the following manner:

$$
\begin{aligned}
& \mathcal{S}_{2}= \mathcal{A} \cap \mathcal{B} \\
& \mathcal{A}=\left\{\left(p_{1}, p_{2}\right) \in \mathbb{R}^{2} \mid f_{1}\left(p_{1}\right) \leq p_{2},\right. \\
&\left.f_{2}\left(p_{1}\right) \geq p_{2}\right\} \\
& \mathcal{B}=\left[0, \frac{P_{1}^{\text {peak }}}{g_{1}}\right] \times\left[0, \frac{P_{2}^{\text {peak }}}{g_{2}}\right] .
\end{aligned}
$$

The set $\mathcal{B}$ represents simply the peak interference constraints in each band which restricts the feasible set $\mathcal{S}_{2}$. Notice that the set $\mathcal{B}$ is not void, however the set $\mathcal{A}$ can be void.

In order to find the conditions under which the set $\mathcal{S}_{2}$ is void or not, i.e., a solution exists or not, we have first to discuss the set $\mathcal{A}$. Thus, we analyse the feasible set $\mathcal{A}$ in the plane $p_{1} \mathcal{O} p_{2}$, where $\mathcal{O}$ is the origin point of coordinates $(0,0)$ and $p_{1}$ and $p_{2}$ are the axes of our plane. In order to do that, we investigate the intersection points $\left(p_{1}, p_{2}\right) \in \mathcal{A}$, of the functions $f_{1}($.$) and$ $f_{2}($.$) by solving the system of equations:$

$$
\left\{\begin{array}{l}
p_{2}=\frac{1}{c_{2}}\left(\frac{\exp \left(R_{\text {min }}\right)}{1+c_{1} p_{1}}-1\right), \\
p_{2}=\frac{\bar{P}-g_{1} p_{1}}{g_{2}}, \\
p_{1} \in\left[0, \frac{P_{1}^{\text {peak }}}{g_{1}}\right],
\end{array}\right.
$$

We further have:

$$
\begin{aligned}
& \left\{\begin{array}{l}
\frac{\bar{P}-g_{1} p_{1}}{g_{2}}=\left(\frac{1}{c_{2}}\right)\left(\frac{\exp \left(R_{\text {min }}\right)}{1+c_{1} p_{1}}-1\right), \\
p_{1} \in\left[0, \frac{P_{1}^{\text {peak }}}{g_{1}}\right], \\
\Leftrightarrow\left(\frac{\bar{P}}{g_{2}}-\frac{g_{1}}{g_{2}} p_{1}\right)=\frac{1}{c_{2}} \frac{\exp \left(R_{\text {min }}\right)}{\left(1+c_{1} p_{1}\right)}-\frac{1}{c_{2}},
\end{array}\right. \\
& \Leftrightarrow\left(\bar{P} c_{2}-g_{1} c_{2} p_{1}+g_{2}\right)\left(1+c_{1} p_{1}\right)=g_{2} \exp \left(R_{\text {min }}\right) .
\end{aligned}
$$

We obtain a quadratic equation w.r.t $p_{1}$

$$
\begin{array}{r}
-g_{1} c_{2} c_{1} p_{1}^{2}+\left(\bar{P} c_{1} c_{2}-g_{1} c_{2}+g_{2} c_{1}\right) p_{1} \\
+\bar{P} c_{2}+g_{2}\left(1-\exp \left(R_{\text {min }}\right)\right)=0 .
\end{array}
$$




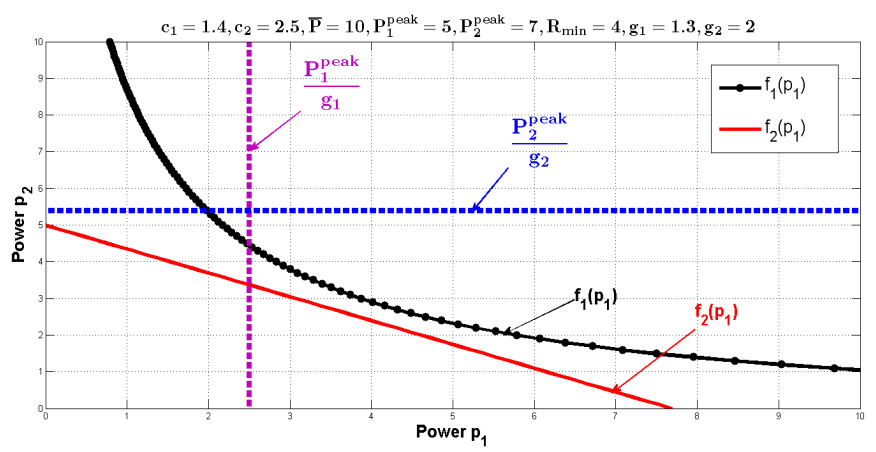

Fig. 2. No Solution because the set $\mathcal{A}=\varnothing \Rightarrow \mathcal{S}_{2}=\varnothing$

Thus, we obtain in (24) a quadratic equation where its discriminant is given by

$$
\begin{gathered}
\Delta_{p_{1}}=\left(\bar{P} c_{1} c_{2}-g_{1} c_{2}+g_{2} c_{1}\right)^{2} \\
+4 g_{1} c_{2} c_{1}\left(\bar{P} c_{2}+g_{2}-g_{2} \exp \left(R_{\text {min }}\right)\right) .
\end{gathered}
$$

(a) If $\Delta_{p_{1}}<0$ we have that $f_{1}\left(p_{1}\right)>f_{2}\left(p_{1}\right) \forall p_{1} \in \mathbb{R}$, this means that the functions $f_{1}($.$) and f_{2}($.$) do not intersect$ i.e the two functions in this case are disjoint (Fig.2) and the set $\mathcal{A}=\varnothing$. Thus, $\mathcal{S}_{2}=\varnothing$ and we have no solution.

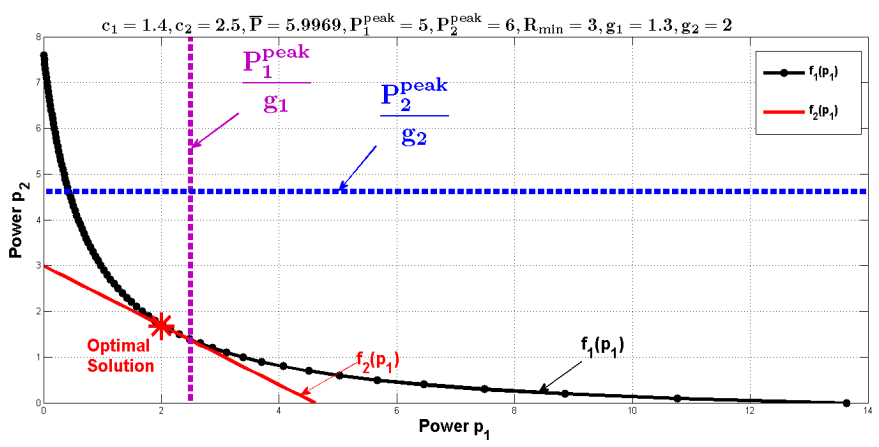

(a) One Solution: $\mathcal{S}_{2}=\left\{\left(p_{1}^{*}, p_{2}^{*}\right)\right\}$.

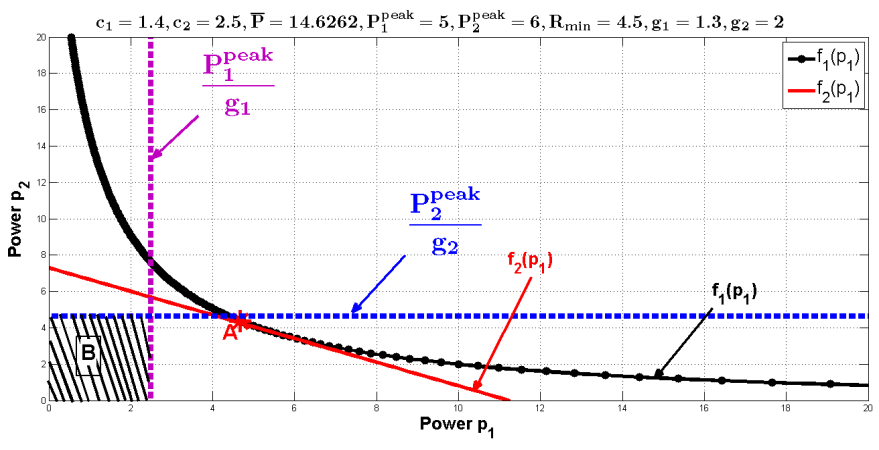

(b) No Solution because $\mathcal{A}=\left\{\left(\widehat{p_{1}}, \widehat{p_{2}}\right)\right\} \cap \mathcal{B}=\varnothing$

Fig. 3. $\Delta_{p_{1}}=0$ and the function $f_{2}($.$) is tangent to the function f_{1}($. where (a) : One Solution, (b): No Solution

(b) If $\Delta_{p_{1}}=0$, then another equation in the second degree is required. To find $\bar{P}$ in this case we calculate the discriminant $\Delta_{\bar{P}}$, which is given by

$$
\Delta_{\bar{P}}=16 c_{1}^{3} c_{2}^{3} g_{1} g_{2} \exp \left(R_{\min }\right)>0 .
$$

Thus, we obtain two solutions:

$$
\bar{P}_{1,2}=-\frac{g_{1}}{c_{1}}-\frac{g_{2}}{c_{2}} \pm 2 \sqrt{\frac{g_{1} g_{2}}{c_{1} c_{2}} \exp \left(R_{\text {min }}\right)} .
$$

Note that one of the two solutions is negative, so it is rejected. Thus, the constraint of average interference powers must satisfy this condition:

$$
\bar{P}=-\frac{g_{1}}{c_{1}}-\frac{g_{2}}{c_{2}}+2 \sqrt{\frac{g_{1} g_{2}}{c_{1} c_{2}} \exp \left(R_{\text {min }}\right)} .
$$

Thus the intersection point in this case $\left(\Delta_{p_{1}}=0\right)$ where $f_{1}($.$) is tangent to f_{2}($.$) , is unique \widehat{p}=\widetilde{p}$ with

$$
\left\{\begin{array}{l}
\widehat{p_{1}}=\frac{\bar{P}}{2 g_{1}} \\
\widehat{p_{2}}=\frac{\bar{P}}{2 g_{2}}
\end{array}\right.
$$

Note that in this case $\mathcal{A}=\left\{\left(\widehat{p_{1}}, \widehat{p_{2}}\right)\right\}$ and if this singleton is in $\mathcal{B}$ (Fig.3. (a)), it is the solution of (17) i.e., $\mathcal{S}_{2}=$ $\left\{\left(\widehat{p_{1}}, \widehat{p_{2}}\right)\right\}$ and $p^{*}=\widehat{p}$. However, if $\left\{\left(\widehat{p_{1}}, \widehat{p_{2}}\right)\right\} \notin \mathcal{B}$ see Fig.3.(b), then, we have no solution and $\mathcal{S}_{2}=\varnothing$.

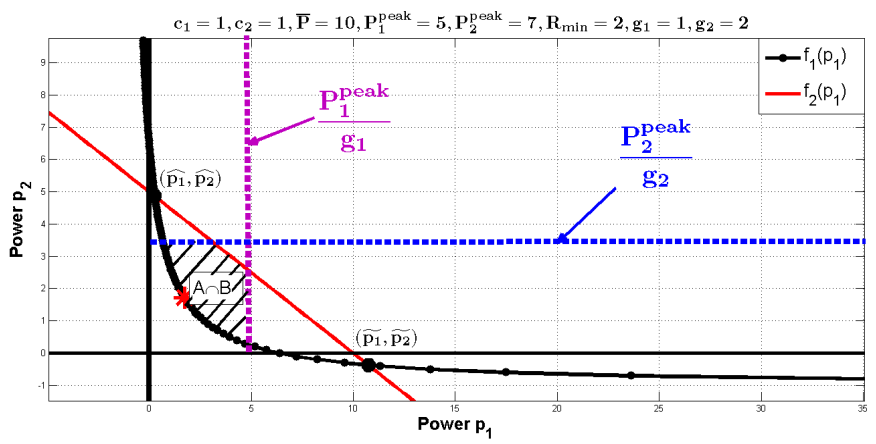

(a) If $\widetilde{p_{1}}<0$ and $\widehat{p_{1}}>0$

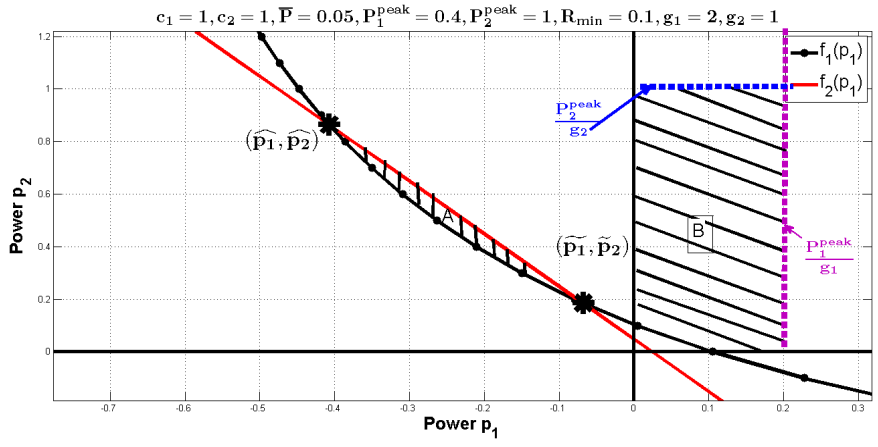

(b) No solution: if $\max \left\{\widehat{p_{1}}, \widetilde{p_{1}}\right\}<0$ because $\mathcal{A} \cap \mathcal{B}=\varnothing$

Fig. 4. Particular cases: either (a): One solution or (b): No solution

(c) If $\Delta_{p_{1}}>0$ which is equivalent to

$\bar{P}>-\frac{g_{1}}{c_{1}}-\frac{g_{2}}{c_{2}}+2 \sqrt{\frac{g_{1} g_{2}}{c_{1} c_{2}} \exp \left(R_{\text {min }}\right)}$, then we have two distinct intersection points (see Fig.4 and Fig.5) $\left(\widehat{p_{1}}, \widehat{p_{2}}\right),\left(\tilde{p_{1}}, \tilde{p_{2}}\right) \in \mathcal{A}$, where 


$$
\begin{aligned}
\widehat{p_{1}} & =\frac{\left(\bar{P} c_{1} c_{2}-g_{1} c_{2}+g_{2} c_{1}\right)-\sqrt{\Delta_{p_{1}}}}{2 g_{1} c_{1} c_{2}}, \\
\widetilde{p_{1}} & =\frac{\left(\bar{P} c_{1} c_{2}-g_{1} c_{2}+g_{2} c_{1}\right)+\sqrt{\Delta_{p_{1}}}}{2 g_{1} c_{1} c_{2}},
\end{aligned}
$$

where $\Delta_{p_{1}}$ is expressed in $(25), \widehat{p_{1}}<\tilde{p_{1}}, \widehat{p_{2}}=f_{1}\left(\widehat{p_{1}}\right)$ and $\tilde{p_{2}}=f_{1}\left(\tilde{p_{1}}\right)$.

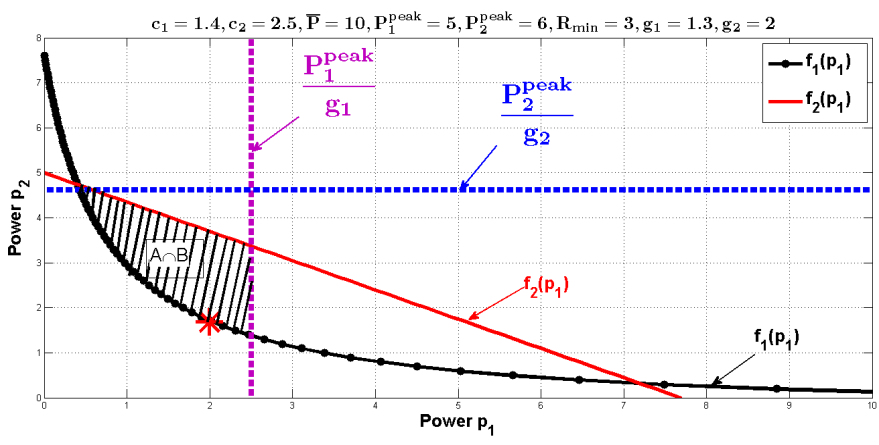

(a) One Solution: if $\mathcal{A} \cap \mathcal{B} \neq \varnothing$

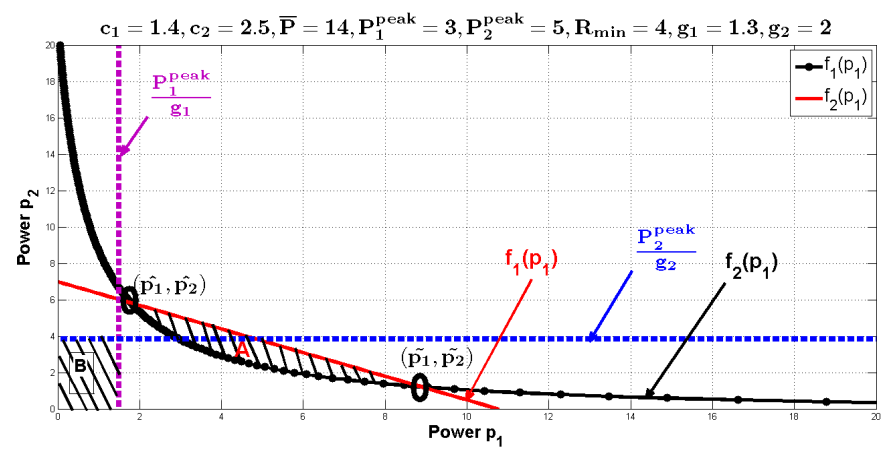

(b) No solution because $\mathcal{A} \cap \mathcal{B}=\varnothing$

Fig. 5. $\quad \Delta_{p_{1}}>0, \mathcal{A} \neq \varnothing$ either (a):One Solution, or (b): No Solution

The discussion in this case is done as a function of the sign of the intersection points $\left(\widehat{p_{1}}, \widehat{p_{2}}\right)$ and $\left(\widetilde{p_{1}}, \widetilde{p_{2}}\right)$.

- If $\max \left\{\widehat{p_{1}}, \widetilde{p_{1}}\right\}<0$ (see Fig.4(b)), then we are back to the case $f_{1}\left(p_{1}\right)>f_{2}\left(p_{1}\right), \forall p_{1} \in \mathbb{R}$. Thus, $\mathcal{S}_{2}=\varnothing$ and we have no solution.

- If $\widehat{p_{1}}$ or $\widetilde{p_{1}}$ is negative, (see Fig.4 (a) when $\widetilde{p_{1}}<0$ ), then we have an unique solution if $\mathcal{A} \cap \mathcal{B} \neq \varnothing$. In this case, see Fig.4(a), the optimal solution $\left(p_{1}^{*}, p_{2}^{*}\right)$ of the problem (17) is not between the intersection points $\left(\widehat{p_{1}}, \widehat{p_{2}}\right)$ and $\left(\widetilde{p_{1}}, \widetilde{p_{2}}\right)$, but it is computed between $\left(\widehat{p_{1}}, \widehat{p_{2}}\right)$ and $\left(0, f_{1}(0)\right)$. These two last cases (if $\max \left\{\widehat{p_{1}}, \widetilde{p_{1}}\right\}<0$ and if $\widehat{p_{1}}$ or $\widetilde{p_{1}}$ is negative) are met, only for small values of $R_{\text {min }}$.

- If both of the two abscissas of the intersection points $\widehat{p_{1}}$ and $\widetilde{p_{1}}$ are positive, (see Fig.5), then we have an unique solution if $\mathcal{A} \cap \mathcal{B} \neq \varnothing$ (see Fig.5(a)) and in this case, the optimal solution $\left(p_{1}^{*}, p_{2}^{*}\right)$ exists. However, if $\mathcal{A} \cap \mathcal{B}=\varnothing$, see Fig.5(b), because of the peak interference constraint $P_{k}^{p e a k}$, then we have no solution.

We give a short sketch of this analysis in Summary 1.

Summary 1 Closed-form analytical solution for the $N=2$ case

If $\bar{P}<-\frac{g_{1}}{c_{1}}-\frac{g_{2}}{c_{2}}+2 \sqrt{\frac{g_{1}}{c_{1}} \frac{g_{2}}{c_{2}} \exp \left(R_{\text {min }}\right)}, \quad$ then $\mathcal{A}=\varnothing$ :

No Solution: Average interference power $\bar{P}$ and $Q o S$ constraints are never simultaneously satisfied

Else $\mathcal{A} \neq \varnothing$,

$$
\text { If } \max \left\{\widehat{p_{1}}, \widetilde{p_{1}}\right\}<0 \Leftrightarrow \mathcal{A} \subset(-\infty, 0] \times[0,+\infty) \text {, }
$$
then $\mathcal{A} \cap \mathcal{B}=\varnothing$ :

No Solution: Average interference power $\bar{P}$ and $Q o S$ constraints are not simultaneously satisfied in $\mathcal{B}$

Else $\max \left\{\widehat{p_{1}}, \widetilde{p_{1}}\right\}>0 \Leftrightarrow \mathcal{A} \not \subset(-\infty, 0] \times[0,+\infty)$,

$$
\begin{aligned}
& \text { If } \frac{P_{1}^{\text {peak }}}{g_{1}}<\widehat{p_{1}} \quad \text { or } \frac{P_{2}^{\text {peak }}}{g_{2}}< \\
& \min \left\{\widehat{p_{2}}, \tilde{p_{2}}\right\} \quad \text { or } \quad\left\{f_{1}\left(\frac{P_{1}^{\text {peak }}}{g_{1}}\right)>\frac{P_{2}^{\text {peak }}}{g_{2}} \quad\right. \text { and } \\
& \left.f_{1}^{-1}\left(\frac{P_{2}^{\text {peak }}}{g_{2}}\right)>\frac{P_{1}^{\text {peak }}}{g_{1}}\right\}, \quad \text { then } \mathcal{A} \cap \mathcal{B}=\varnothing: \\
& \text { No Solution: } P_{k}^{\text {peak }} \text { constraints are not satisfied }
\end{aligned}
$$

Else $\quad \mathcal{A} \cap \mathcal{B} \neq \varnothing$ :

Exists a solution given by:

$$
\left\{\begin{array}{l}
p_{1}^{*}=\left[\frac{-1}{c_{1}}+\sqrt{\frac{\exp \left(R_{\text {min }}\right)}{c_{1} c_{2}}}\right]_{x}^{y}, \\
p_{2}^{*}=\left[\frac{-1}{c_{2}}+\sqrt{\frac{\exp \left(R_{\text {min }}\right)}{c_{1} c_{2}}}\right]_{w}^{z} .
\end{array}\right.
$$

\section{EndIf}

\section{EndIf}

\section{EndIf}

where $x, y, w, z$ are given in Theorem 2 in equation (18), and $\left(\widehat{p_{1}}, \widetilde{p_{2}}\right),\left(\widetilde{p_{1}}, \widetilde{p_{2}}\right)$ are given in (30) and $\Delta_{p_{1}}$ is given in (25). 


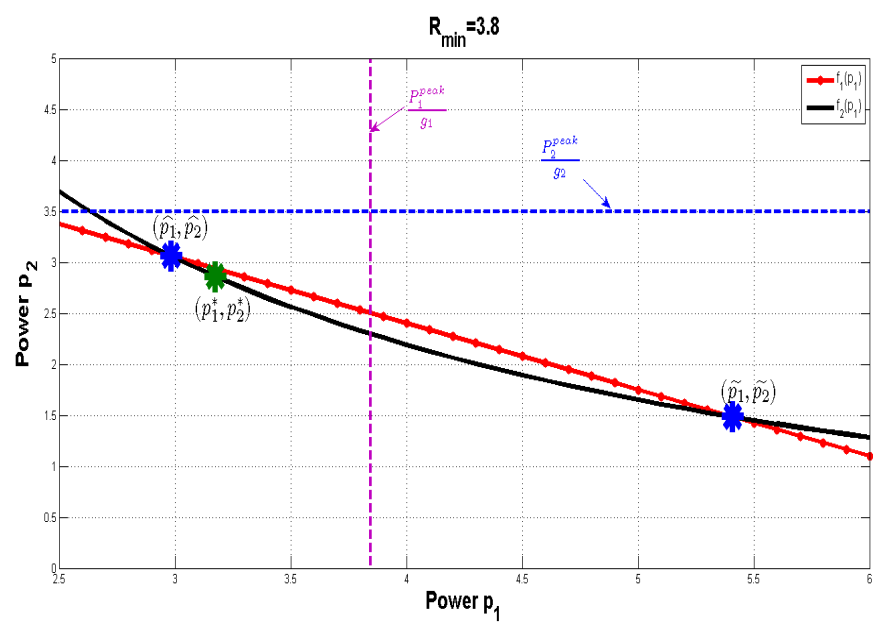

(a) One solution $\left(p_{1}^{*}, p_{2}^{*}\right)$ gets closer to $\left(\widehat{p_{1}}, \widehat{p_{2}}\right)$

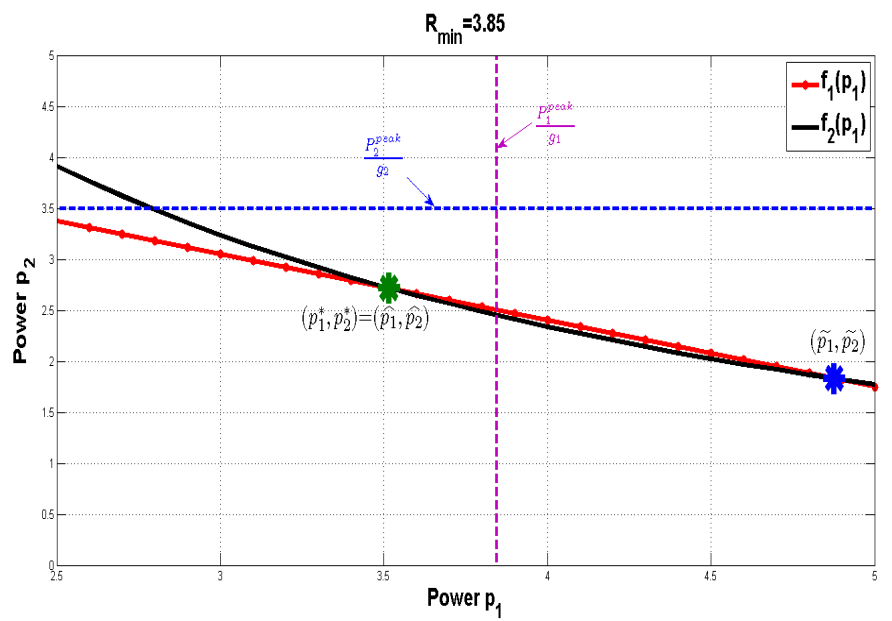

(b) One solution $\left(p_{1}^{*}, p_{2}^{*}\right)=\left(\widehat{p_{1}}, \widehat{p_{2}}\right)$

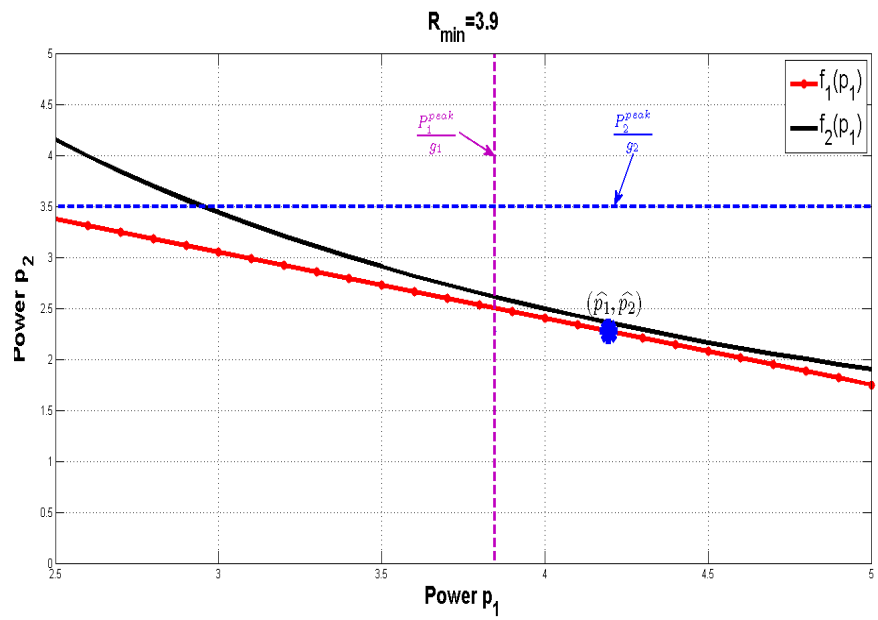

(c) No solution $\left(p_{1}^{*}, p_{2}^{*}\right)$ gets out of the set $\mathcal{B}$

Fig. 6. The average interference constraint is satisfied with a strict inequality in ?? and (a), or with equality in (b). However in (c) the feasible set does not exist

\section{NUMERICAL SIMULATIONS}

Our simulation parameters are generic and, thus, all the observations we make here under remain valid for any values of the parameters and any practical system.

A. SU Rate and Interference Powers Caused by SU to PU vs. QoS Constraint $R_{\text {min }}$

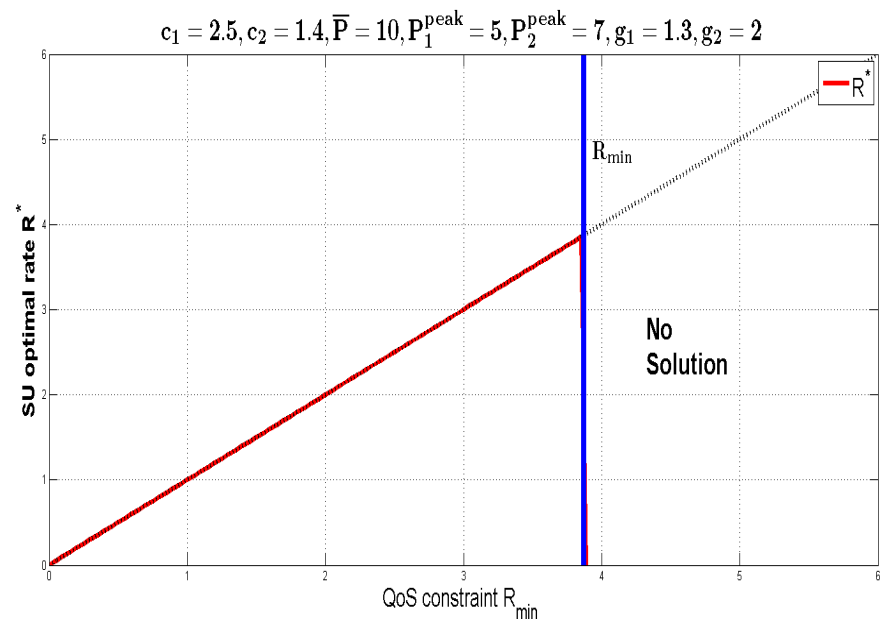

(a) SU optimal rate vs. rate constraint $R_{\min }$

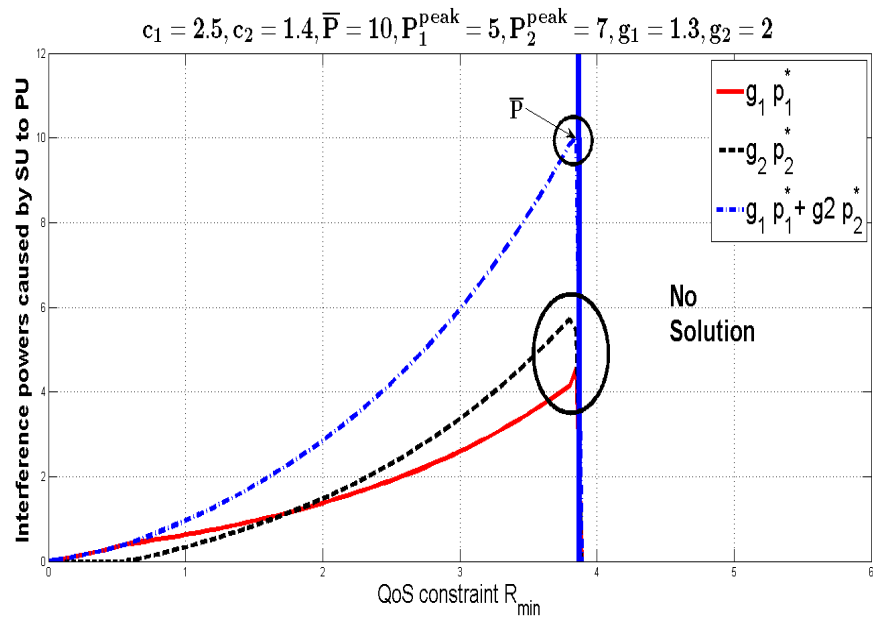

(b) Interference levels caused by SU to PU vs. rate constraint $R_{\text {min }}$

Fig. 7. SU optimal rate (a) and interference levels caused by SU to PU (b) vs. rate constraint $R_{\text {min }}$

In Fig.7, we plot the SU optimal rate $R^{*} \triangleq R\left(\underline{p}^{*}\right)$ defined in (2)) and the interference powers levels caused by $\mathrm{SU}$ to PU $\left(g_{1} p_{1}^{*}, g_{2} p_{2}^{*}\right.$ and $\left.g_{1} p_{1}^{*}+g_{2} p_{2}^{*}\right)$ versus the minimum rate constraint $R_{\text {min }}$ over two orthogonal frequency channels with the system parameters: $c_{1}=2.5, c_{2}=1.4, \bar{P}=10, P_{1}^{\text {peak }}=$ $5, P_{2}^{\text {peak }}=7, g_{1}=2$ and $g_{2}=1.3$.

In the two sub-figures, we observe that there is a zone where our problem does not admit a solution where the average interference power and QoS constraints are not simultaneously satisfied (for example the case of Fig.2). However, there is a critical value when $R_{\min } \approx 3.866$ below which an optimal solution exists. Therefore, if the QoS constraint is too restrictive, i.e., $R_{\min }$ is above this threshold, a feasible solution does not exist.

In Fig.7.(a), we see that the optimal rate is equal to the minimum rate constraint $R_{\min }$ which is trivial, because of the 
KKT condition of $\lambda>0$ in (15).

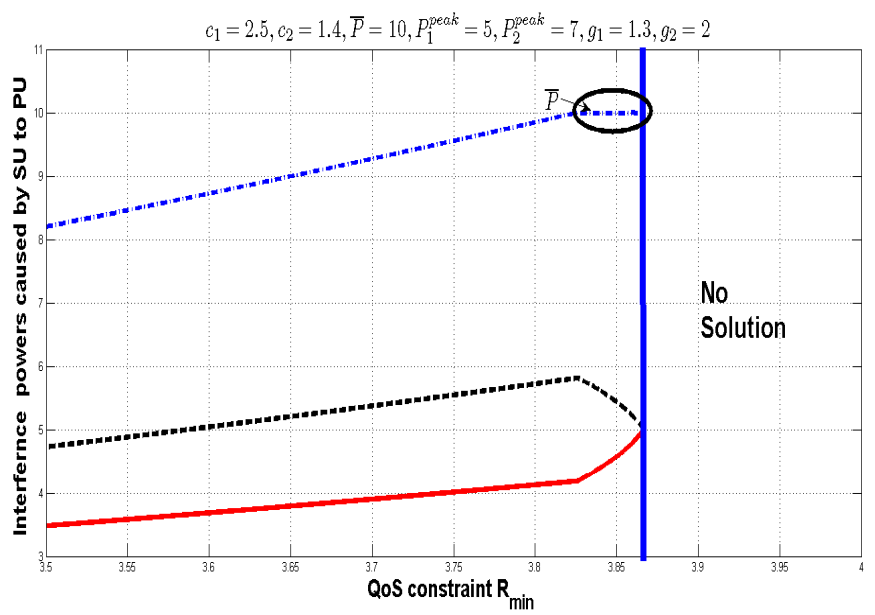

Fig. 8. Zoom of Fig.7 (b) for some values of $R_{\min }$

In Fig.7.(b) zoomed in Fig.8, we remark that in the interval where $R_{\min } \in[0,8.26]$ the average interference constraint is satisfied with a strict inequality $\left(\sum_{k=1}^{N} g_{k} p_{k}^{*}<\bar{P}\right)$ which are illustrated in Fig.6.(a). We observe that the transmit powers required to achieve the QoS constraint $R_{\text {min }}$, increase exponentially. Also the optimal point $\left(p_{1}^{*}, p_{2}^{*}\right)$ is on the curve $f_{1}(p)$ in between $\widehat{p}$ and $\widetilde{p}$. By increasing $R_{\min }$ we observe that $p^{*}$ gets closer to $\widehat{p}$. It turns out that in this scenario, when $R_{\text {min }}>8.26$ the average interference constraint is satisfied with equality and the optimal solution is equal to the intersection point $p^{*}=\widehat{p}$ which is illustrated in Fig.6.(b). Finally when $R_{\min }$ exceeds the critical value of 3.866 then a feasible solution does not exist as we see in Fig.6.(c) because $\underline{\widehat{p}}$ gets out of the set $\mathcal{B}$.

\section{B. SU Rate and Interference Powers Caused by SU to PU vs. Interference Power Constraint $\bar{P}$}

In Fig.9, we plot the SU rate $R^{*}$ and the interference powers levels caused by $\mathrm{SU}$ to $\mathrm{PU}\left(g_{1} p_{1}^{*}, g_{2} p_{2}^{*}\right.$ and $\left.g_{1} p_{1}^{*}+g_{2} p_{2}^{*}\right)$ versus the average interference power $\bar{P}$ over two orthogonal frequency channel with the system parameters: $c_{1}=2.5, c_{2}=$ $1.4, R_{\text {min }}=3, P_{1}^{\text {peak }}=5, P_{2}^{\text {peak }}=7, g_{1}=2$ and $g_{2}=1.3$. Similarly to the previous figure there is a zone where there is no feasible solution.

In Fig.9.(a) the $\mathrm{SU}$ rate is equal to the minimum rate constraint $R_{\min }$ for $\bar{P}>6$. In conclusion, if $\bar{P}$ is below a certain threshold then the average interference constraint is too restrictive and a solution does not exist.

In Fig.9.(b) we note that the interference power level caused by SU to PU for each orthogonal frequency band is constant and independent from $\bar{P}$. Therefore, $\bar{P}$ does not influence the expressions of the optimal power $\underline{p}^{*}$ once it exists. For example, if $\bar{P} \simeq 6$, the average interference constraint is satisfied with equality. However, if $\bar{P}>6$ we obtain $g_{1} p_{1}^{*}+g_{2} p_{2}^{*}<\bar{P}$, then the average interference constraint is satisfied with strict inequality.

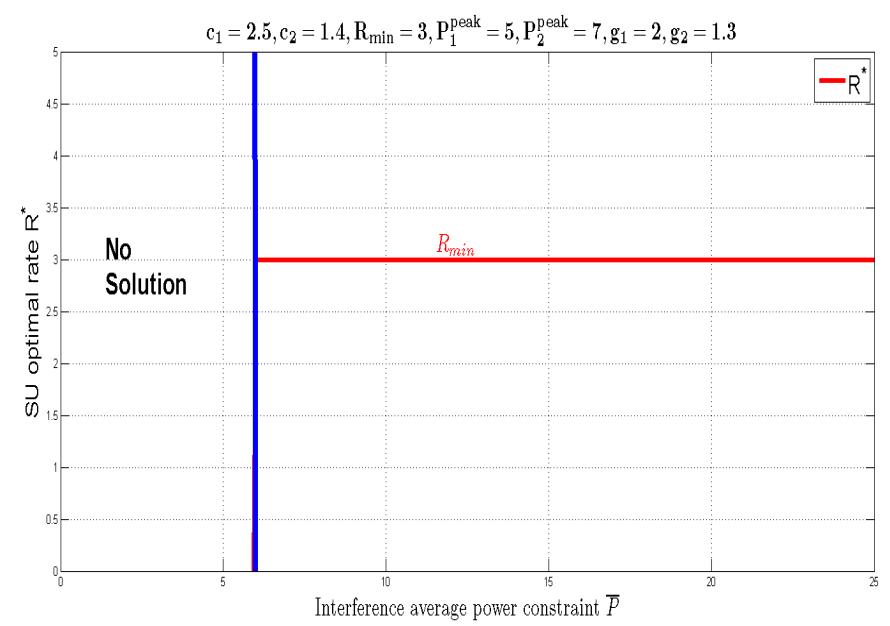

(a) SU optimal rate vs. interference power constraint $\bar{P}$

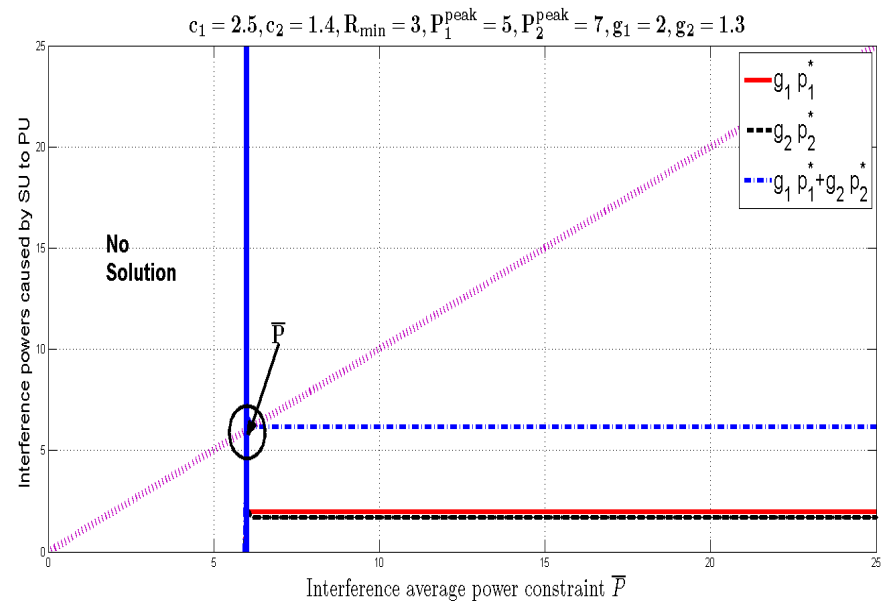

(b) Interference levels caused by SU to PU vs. interference power constraint $\bar{P}$

Fig. 9. SU optimal rate (a) and interference levels caused by SU to PU (b) vs. interference power constraint $\bar{P}$

\section{SU Rate and Interference Powers Caused by SU to PU vs. the Direct Link Gain $c_{1}$}

In Fig.10, we plot the SU rate $R^{*}$ and the interference power level caused by SU to PU $\left(g_{1} p_{1}^{*}, g_{2} p_{2}^{*}\right.$ and $\left.g_{1} p_{1}^{*}+g_{2} p_{2}^{*}\right)$ versus the direct link gain in the first band $c_{1}$ in the scenario: $c_{2}=1.4, R_{\min }=3, \bar{P}=10, P_{1}^{\text {peak }}=5, P_{2}^{\text {peak }}=7$, $g_{1}=2$ and $g_{2}=1.3$.

In Fig.10.(a), the optimal rate is equal to the minimum rate constraint $R_{\min }$ for all $c_{1}$ values $\left(\forall c_{1}>0.9\right)$. We see that if the quality of one of the direct links (i.e., small values of the direct link gain $c_{1}$ ) is very poor, then the QoS constraint cannot be satisfy and thus, a feasible solution does not exist.

In Fig.10.(b), we note that the optimal power for each orthogonal frequency band decreases exponentially with the direct link gain $c_{1}$. The higher the quality of the direct links, the lower are the powers required to achieve the QoS constraint. For example, if $c_{1} \simeq 0.9$, we see that the average interference constraint is satisfied with equality. However, if $c_{1}>0.9$ the average interference constraint is satisfied with 


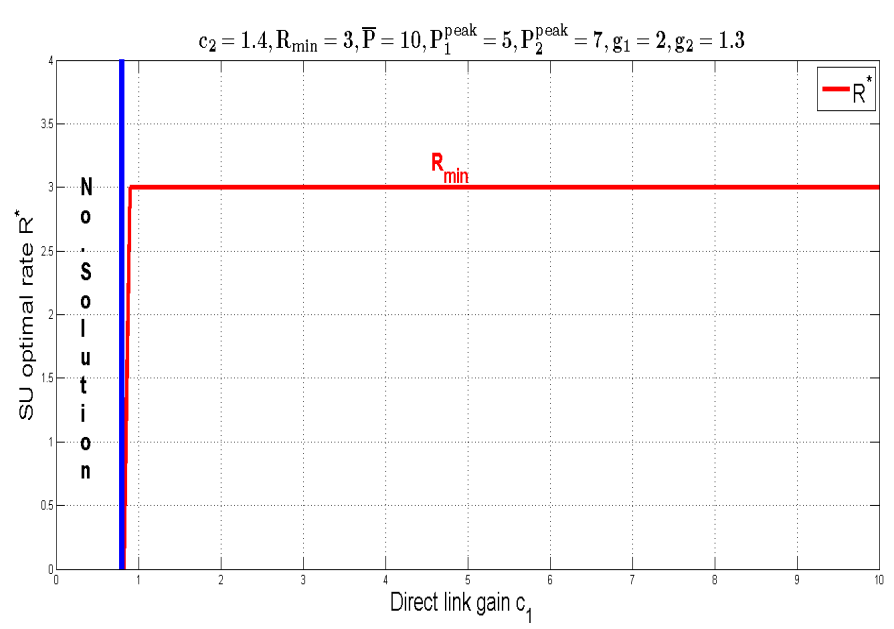

(a) SU optimal rate vs. the direct link gain $c_{1}$

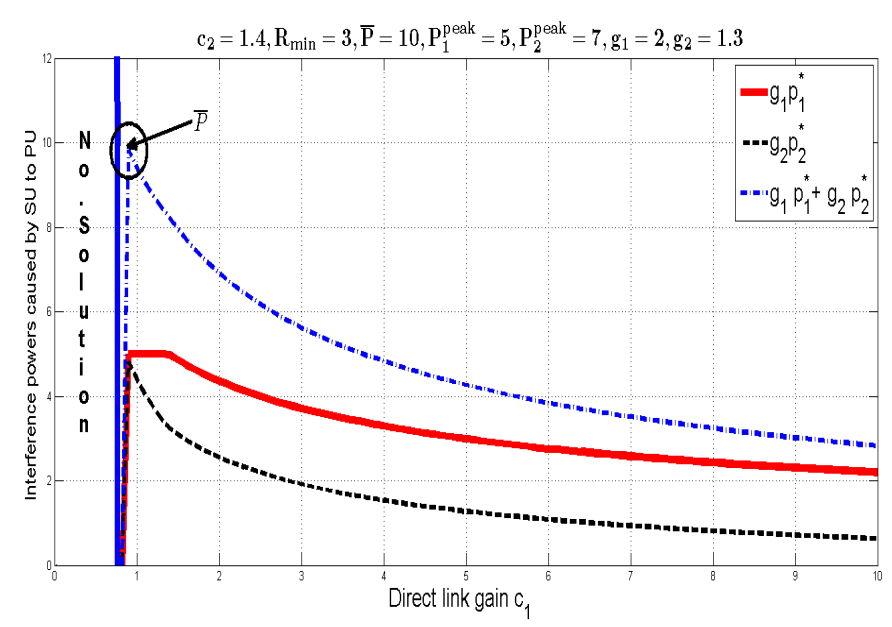

(b) Interference levels caused by SU to PU vs. the direct link gain $c_{1}$

Fig. 10. SU optimal rate (a) and interference levels caused by SU to PU (b) vs. the direct link gain $c_{1}$

strict inequality i.e. $g_{1} p_{1}^{*}+g_{2} p_{2}^{*}<\bar{P}$.

\section{SU Rate and Interference Powers Caused by SU to PU vs. the interference link gain $g_{1}$}

In Fig.11, we plot the optimal rate $R^{*}$ and SU rate and interference powers caused by SU to PU $\left(g_{1} p_{1}^{*}, g_{2} p_{2}^{*}\right.$ and $\left.g_{1} p_{1}^{*}+g_{2} p_{2}^{*}\right)$ versus the interference link gain $g_{1}$ over two orthogonal frequency channels with the system parameters: $c_{1}=2.5, c_{2}=1.4, R_{\min }=4, P_{1}^{\text {peak }}=5, P_{2}^{\text {peak }}=7$, $\bar{P}=10$ and $g_{2}=1.3$.

In Fig.11.(a), the optimal rate is equal to the minimum rate constraint $R_{\text {min }}$ for values of the interference link gain $g_{1}$ below a certain threshold. We see that when the quality of the interfering link is high (i.e. high values of $g_{1}$ ) the maximal interference constraint are not met and a feasible solution does not exist.

In Fig.11.(b), we observe that, except for some borders values (nearly the critical threshold) where $p_{1}^{*}=\frac{P_{1}^{\text {peak }}}{g_{1}}$ (see equation (18)) the optimal transmit power value $p_{1}^{*}$ does not

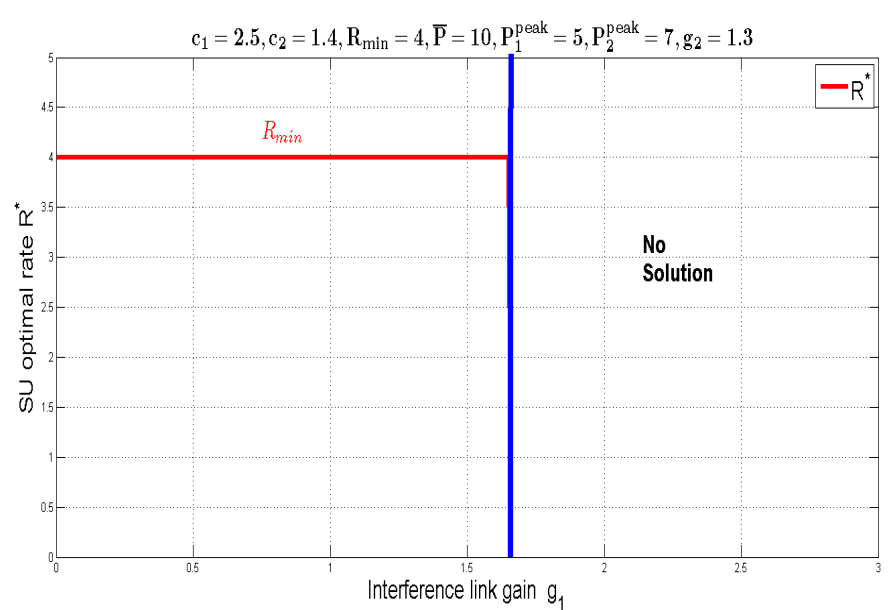

(a) SU optimal rate vs. the interference link gain $g_{1}$

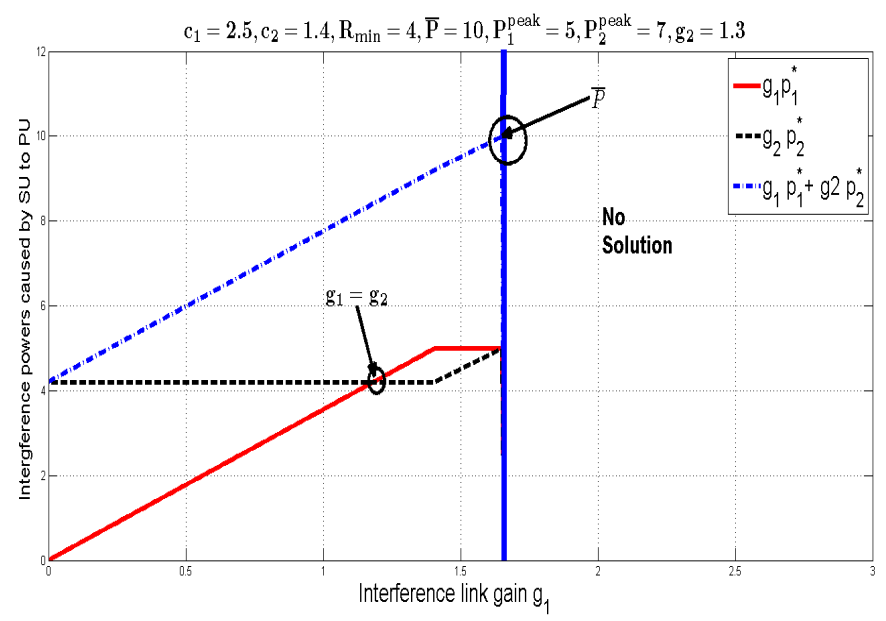

(b) Interference levels caused by SU to PU vs. the interference link gain $g_{1}$

Fig. 11. SU optimal rate (b) and interference levels caused by SU to PU (b) vs. the interference link gain $g_{1}$

depend on $g_{1}$. The optimal transmit power $p_{2}^{*}$ is independent of the interference link gain $g_{1}$ except for the same region where $p_{2}^{*}$ is proportional to the interference link gain $g_{1}$ (see equation (18)). For example, if $g_{1} \simeq 1.7$, we see that the average interference constraint is satisfied with equality. However, if $c_{1}<1.7$ the average interference constraint is satisfied with strict inequality i.e. $g_{1} p_{1}^{*}+g_{2} p_{2}^{*}<\bar{P}$.

In the case where $N>2$, the difficulty lies in the fact that the iterative water-filling algorithm converges to the optimal solution provided that the feasibility set of all the constraints is non void. Finding the conditions on the system parameters that ensure that the feasible set is non void, is a difficult problem since the number of constraints and the degrees of freedom becomes larger.

\section{Conclusions}

In this paper, we have analysed the power allocation problem over orthogonal bands at the opportunistic user's level 
under a target QoS constraint and maximal interference caused to the primary user of the spectrum constraints.

We have seen that, depending on the system parameters, a feasible solution does not always exists. This is mainly caused by the simultaneous requirements of minimum target achievable rate at the $\mathrm{SU}$ and maximum allowed interference level caused to the PU. When a solution exist, it takes the form of water-filling and the closed-form solution is provided for the particular case of two available frequency bands.

Future work include the case of multiple secondary and primary users for which efficient distributed algorithms have to be proposed. Also, we will consider the effect of imperfect parameters estimation or knowledge, and investigate the robustness of our power allocation problem to these imperfections.

\section{REFERENCES}

[1] J. Mitola, "Cognitive radio architecture evolution," IEEE Trans. Inf. Theory, vol. 97, no. 4, pp. 626-641, Apr. 2009.

[2] E. V. Belmega and S. Lasaulce, "An information-theoretic look at MIMO energy-efcient communications," in International Conference on Performance Evaluation Methodologies and Tools (VALUETOOLS), Pisa, Italy, Oct. 2009

[3] E. V. Belmega, S. Lasaulce, and M. Debbah, "A survey on energyefficient communications," in IEEE Intl. Symp. on Personal, Indoor and Mobile Radio Communications (PIMRC), Istanbul, Turkey, Sep. 2010.

[4] R. Cendrillon, W. Yu, M. Moonen, J. Verlinder, and T. Bostoen, "Optimal multi-user spectrum managment for digital subscriber lines," vol. 54, no. 5, pp. 922-933, May. 2006.

[5] G. Scutari, D. P. Palomar, and S. Barbarossa, "Asynchronous iterative water- filling for gaussian frequency-selective interference channels," IEEE Trans. Inf. Theory, vol. 54, no. 7, pp. 2868-2878, Jul. 2008.

[6] A. Bagayoko, I. Fijalkow, and P. Tortelier, "Power control of spectrumsharing in fading environment with partial channel state information," IEEE Trans. Signal Process., vol. 59, no. 5, pp. 2244-2256, May 2011.

[7] M. S. Bazaraa, H. D. Sherali, and C. M. Shetty, Nonlinear programmingTheory and Algorithms. New York: Wiley, 1993.

[8] S.Boyd and L. Vandenberghe, Convex Optimization. Combridge , UK: Combridge Univ. Press, 2004.

[9] G. Scutari and D. P. Palomar, Competitive Optimization of Cognitive Radio MIMO Systems via Game Theory. Combridge, UK: Combridge Univ. Press, 2009.

[10] J.-S. Pang, G. Scutari, F. Facchinei, and C. Wang, "Distributed power allocation with rate constraints in gaussian parallel interference channels," IEEE Trans. Inf. Theory, vol. 54, no. 8, pp. 3471-3489, Aug. 2008. 